Vol. $7, n^{\circ} 1 \mid 2003$

Varia

\title{
La criminalité de sang en Corse sous la domination génoise (fin $\mathrm{XVII}^{\mathrm{e}}$-début $\mathrm{XVIII}^{\mathrm{e}}$ siècles)
}

\section{Antoine Laurent Serpentini}

\section{(2) OpenEdition}

\section{Journals}

Édition électronique

URL : https://journals.openedition.org/chs/607

DOI : $10.4000 /$ chs. 607

ISSN : 1663-4837

Éditeur

Librairie Droz

\section{Édition imprimée}

Date de publication : 1 juillet 2003

Pagination : $57-78$

ISBN : 2-600-00865-9

ISSN : $1422-0857$

\section{Référence électronique}

Antoine Laurent Serpentini, «La criminalité de sang en Corse sous la domination génoise (fin XVII edébut XVIII siècles) », Crime, Histoire \& Sociétés / Crime, History \& Societies [En ligne], Vol. 7, $n^{\circ} 1$ | 2003, mis en ligne le 23 février 2009, consulté le 22 mars 2022. URL : http://journals.openedition.org/chs/ 607 ; DOl : https://doi.org/10.4000/chs.607

Ce document a été généré automatiquement le 22 mars 2022.

(c) Droz 


\title{
La criminalité de sang en Corse sous la domination génoise (fin XVII ${ }^{\mathrm{e}}$ début XVIII ${ }^{\mathrm{e}}$ siècles)
}

\author{
Antoine Laurent Serpentini
}

\section{L'impact réel de la criminalité de sang}

1 En 1758, dans un très virulent pamphlet visant à légitimer la révolte des Corses contre la République de Gênes, l'abbé Don Gregorio Salvini'2 l'un des conseillers les plus écoutés de Pascal Paoli, dénonçait, entre autres tares, l'impuissance flagrante de la justice génoise à juguler le banditisme dans l'île, et plus particulièrement la criminalité de sang qui semblait avoir pris des proportions terrifiantes. Salvini, reprenant et développant les arguments avancés, quelque vingt ans plus tôt, par le chanoine Natali ${ }^{3}$ affirmait en substance que si Gênes avait finalement pris la décision d'interdire les armes à feu en $1715^{3}$, ce fut après examen des registres de criminalité tenus par les chancelleries des dix provinces insulaires d'où il ressortait que la population, déjà éprouvée par tant de maux, avait eu à subir 28715 homicides durant les trente-deux années précédentes.

228715 meurtres entre $168^{4}$ et 1715 , soit environ 900 meurtres par an (897 exactement) pour une population qui, durant la même période, oscille entre 120000 et 130000 âmes ${ }^{5}$. Ces données posent incontestablement problème et, pour le moins, auraient dû interpeller avant ce jour les historiens et les démographes. En effet, si on avalise ces chiffres, il faudrait admettre que pendant cette période quasiment toutes les familles insulaires ont participé de cette violence sanguinaire ou bien ont eu à la subir à travers au moins un de leurs membres. Par ailleurs, si on les reporte à la France dont la population, pour la même période, est estimée à 23 millions d'habitants ${ }^{6}$, on obtient environ 158000 homicides par an et 500000 pour les trente-deux années considérées, c'est-à-dire - toutes proportions gardées - une ponction bien plus importante que celle subie par l'Europe belligérante durant la guerre de Trente ans, et qui se rapprocherait, 
comme le souligne Emmanuel Le Roy Ladurie ${ }^{7}$, de celle que provoqua dans l'hexagone la guerre de 1914-1918 ! On pourrait aisément nous objecter que l'histoire de la Corse ne se confond pas, à cette époque, avec celle de la France et que les taux de criminalité de l'île ne sauraient être comparés à ceux d'un royaume en expansion, incontestablement plus policé et à la démographie plus vigoureuse. Certes, et nous en convenons d'autant plus volontiers que nous récusons les estimations avancées par Salvini.

Il n'est pas possible d'aller vers des projections fiables en l'absence d'indications précises concernant et la structure de la population insulaire et l'âge moyen des victimes. Cependant, les renseignements dont nous disposons nous permettent d'affirmer, d'une part, que le prélèvement portait massivement sur la population masculine ${ }^{8}$, vraisemblablement très majoritairement comprise dans les tranches d'âge 20-60 ans, et que, d'autre part, la population insulaire croît de façon assez sensible à partir de la fin du XVII et que le mouvement est soutenu jusqu'aux années $1770^{9}$.

4 Il semble donc évident que la criminalité de sang n'a jamais pu atteindre de tels niveaux. Pourtant, tous ceux qui depuis se sont intéressés à cet aspect du passé insulaire, à commencer par le génois Francesco Maria Acinelli ${ }^{10}$ dans les années 1770, ont accrédité ces informations ${ }^{11}$, avant tout soucieux de mettre en exergue les effets destructeurs de l'incurie du système génois ou bien ceux des mœurs présupposés sanguinaires des Corses, voire de la conjonction de ces deux phénomènes. Seul Emmanuel Le Roy Ladurie, bien que n'étant pas en mesure de vérifier ces affirmations, s'en étonne et parle à ce propos d'île " ethnographiquement violente ${ }^{12}$. La sévérité de l'appréciation a été relevée par certains historiens insulaires ${ }^{13}$, elle nous semble pourtant justifiée s'appliquant à ce que l'on pensait être la réalité criminogène du temps. Heureusement pour l'île, cette criminalité de sang, bien qu'étant incontestablement une des plus fortes d'Europe, n'était cependant en rien comparable à cette véritable vision d'apocalypse évoquée par Natali et Salvini. Nous sommes en mesure, aujourd'hui, d'en apporter la preuve.

Partant des assertions mêmes de l'abbé Salvini, nous nous sommes efforcés de retrouver dans les archives génoises les fameux registres en question. Nombre d'entre eux reposaient, comme c'était prévisible, à l'Archivio di Stato de Gênes et, plus précisément, dans les liasses de la série Litterarum du Fonds Corsica. Ils ne recouvrent pas parfaitement la période ci-dessus définie, mais ils sont assez nombreux et surtout suffisamment riches d'informations diverses pour pouvoir témoigner de la situation. Il faut souligner leur intérêt, car il est rare que les archives judiciaires des Temps Modernes fournissent un corpus d'une telle richesse couvrant un espace aussi vaste sur un temps relativement long ${ }^{14}$. Nous en livrons ici les premiers enseignements.

6 Ces documents se présentent sous forme de rapports adressés, en fin de mandat, par chaque gouverneur de l'île au Magistrato ou Ufficio di Corsica, organisme qui, à Gênes, coiffait la gestion de la Corse. Ainsi le plus ancien, daté du 25 avril 1692, comptabilise les crimes commis dans les années 1690-1692, sous le gouvernement de Giovanni Prato ${ }^{15}$. Pour les années 1692-1694, seul a été conservé le relevé concernant la province de Bastia ${ }^{16}$. Le rapport suivant nous fait remonter au 24 du mois de juin 1702, date à laquelle le gouverneur Filippo Adorno entre en fonction. Il énumère les homicides commis jusqu'au 18 juin 1704, au moment où le gouverneur, arrivé au terme de son mandat de deux ans (biennio), s'apprête à quitter l'île ${ }^{17}$. Faisant suite à ceci, une belle série de rapports bi-annuels, avec une seule interruption (années 1708-1710), nous conduit jusqu'en $1720^{18}$. Enfin, un dernier rapport couvre partiellement les années 
1726-1728 ${ }^{19}$. Il recense uniquement quatorze assassinats et sa présentation ainsi que l'absence d'autres états pour les années précédentes nous amènent à penser qu'à une époque où bien d'autres dangers se précisent, la recension des homicides n'est plus pour Gênes une priorité. Nous n'avons donc pas tenu compte de ce dernier registre pour l'élaboration de nos statistiques.

7 De la lecture des diverses relations retenues, il ressort tout d'abord que les gouverneurs de l'île arrivés au terme de leur mandat et avant que de retourner dans la métropole, étaient effectivement tenus de fournir aux autorités de tutelle un décompte des homicides commis en Corse durant leur biennio ${ }^{20}$. Dans ce dessein, ils exigeaient de leurs subordonnés investis de pouvoirs de justice, les commissaires et les lieutenants des neuf provinces de l'île ${ }^{21}$, un compte rendu détaillé des meurtres commis dans leur circonscription administrative durant la période correspondante. Ces rapports qui, tous les deux ans du mois d'avril au mois de juin, remontaient des provinces, vont avec le temps devenir de plus en plus riches en renseignements de toutes sortes ${ }^{22}$. Au début, ils se limitent à dénombrer les meurtres en les répartissant en trois ou quatre rubriques : ceux qui ont été commis intentionnellement (dolosi) et (ou) dans le cadre de vendette transversales, ceux qui sont survenus lors de rixes (rissosi), ceux qui concernent des bandits ou bannis éliminés (banditi estinti) et enfin les décès accidentels (casuali) qui sont apparemment assimilés à des morts violentes et donc soumis à un contrôle judiciaire.

8 Puis, au fur et à mesure que l'on avance dans le XVIII ${ }^{\mathrm{e}}$ siècle, ces documents mentionneront bien souvent la date, les circonstances et la qualification du meurtre, toujours l'identité des victimes, très fréquemment celle des meurtriers et parfois la sanction judiciaire qui s'en est suivie. Ce faisant, malgré la brièveté quasi systématique des informations livrées, ils se révèlent non seulement assez explicites pour nous permettre de cerner avec précision la réalité de la criminalité de sang, mais ils nous fournissent également des renseignements précieux concernant la pratique de la procédure criminelle mise en place pour essayer de la juguler. Il faut dire aussi que les chancelleries des provinces ne tiennent pas toujours une comptabilité séparée des meurtres survenus dans la circonscription. Ceux-ci sont alors enregistrés au jour le jour, au même titre que tous les autres délits, quelle qu'en soit la gravité, précédés d'un numéro qui est souvent répercuté dans le rapport final adressé au gouverneur. Une lecture rapide de ces rapports a peut-être fait confondre le numéro indiquant l'ordre chronologique de l'enregistrement et le nombre réel des meurtres. C'est une éventualité à retenir, étant donné la disproportion existant entre le nombre de meurtres annoncés et ceux réellement commis, ou du moins recensés. Mais il est encore plus vraisemblable que les autorités génoises aient, à un moment donné, volontairement grossi l'impact du phénomène pour frapper l'opinion insulaire et lui faire plus facilement accepter l'interdiction des armes à feu.

9 Même si un certain sous-enregistrement n'est pas à exclure ${ }^{23}$, une première constatation s'impose, la criminalité de sang telle qu'elle se dégage des données décrites supra et présentées dans le tableau $n^{\circ} 1$ est infiniment moins importante que celle qui a été admise jusqu'à présent. Durant les vingt années pour lesquelles nous disposons d'informations ${ }^{24}$ on dénombra 1123 cas, soit 56,2 meurtres par an. Si l'on ne prend en considération que le début du XVIII ${ }^{\mathrm{e}}$ siècle, période pour laquelle les données sont plus suivies et plus fournies, on comptabilise 946 morts violentes sur seize années. Il faut, bien entendu, soustraire de ce total les 78 décès accidentels survenus durant ce 
laps de temps et qui ont été pris en compte, on aboutit ce faisant à 868 meurtres, soit une moyenne annuelle de 54,3 homicides, qui reportée à l'intervalle de trente-deux ans retenu, donnerait 1738 meurtres. Pour une population estimée au minimum à 122000 âmes, on obtient, sur ces bases, un taux annuel de mortalité par homicide s'élevant à $0,045 \%$ ou à 45,3 pour 100000 habitants, ce qui pour l'époque est déjà considérable.

Il appert cependant que les résultats affichés sont très contrastés. Cela va de 223 meurtres, toutes qualifications confondues, pour les années 1704-1706 à 53, voire 29 en fin de période, avec une pointe entre 1702 et 1716 (période durant laquelle la moyenne annuelle plafonne à 72 morts violentes) ou bien, si l'on évacue les décès accidentels, à 66,5 meurtres, soit au pire 2128 homicides en trente-deux ans, ce qui donne un taux annuel de 54,5 pour 100000 habitants.

Tableau $n^{\circ} 1$ - Total des homicides perpétrés dans l'île

\begin{tabular}{|c|c|c|c|c|c|c|c|c|c|c|c|c|c|}
\hline PÉRIODES & \multicolumn{12}{|c|}{ PROVINCES (1) } & \multirow{2}{*}{ ENSEMBLE } \\
\hline & 1 & 2 & 3 & 4 & 5 & 6 & A & 7 & 8 & 9 & 10 & B & \\
\hline $1690-1692$ & 37 & - & 7 & 4 & 7 & 11 & 66 & 25 & 11 & 4 & 4 & 44 & 110 \\
\hline 1692-1694 & 67 & & & & & & 67 & & & & & & 67 \\
\hline 1694-1702 & & & & & & & & & & & & & \\
\hline $1702-1704$ & 27 & 1 & 6 & 16 & 19 & 7 & 76 & 47 & 1 & 13 & 2 & 63 & 139 \\
\hline 1704-1706 & 80 & - & 9 & 14 & 25 & 23 & 151 & 41 & 10 & 18 & 3 & 72 & 223 \\
\hline 1706-1708 & 24 & 2 & 4 & 3 & 14 & 15 & 62 & 19 & 2 & 1 & 1 & 23 & 85 \\
\hline $1708-1710$ & & & & & & & & & & & & & \\
\hline $1710-1712$ & 40 & 3 & 2 & 5 & 16 & 19 & 85 & 42 & - & 14 & 5 & 61 & 146 \\
\hline $1712-1714$ & 34 & 1 & 10 & 5 & 15 & 20 & 85 & 34 & 4 & 12 & 5 & 55 & 140 \\
\hline $1714-1716$ & 33 & 2 & 1 & 11 & 18 & 7 & 72 & 40 & 8 & 6 & 4 & 58 & 130 \\
\hline 1716-1718 & 24 & 5 & 1 & - & 4 & 2 & 36 & 10 & 2 & 3 & 2 & 17 & 53 \\
\hline $1718-1720$ & - & 1 & 3 & - & 11 & 1 & 16 & 6 & - & - & 8 & 14 & 30 \\
\hline $1690-1720$ & 366 & 15 & 43 & 58 & 129 & 105 & 716 & 264 & 38 & 71 & 34 & 407 & 1123 \\
\hline $1702-1720$ & 262 & 15 & 36 & 54 & 122 & 94 & 583 & 239 & 27 & 67 & 31 & 363 & 946 \\
\hline
\end{tabular}

(1) Provinces: 1. Bastia, 2. Rogliano, 3. Calvi, 4. Algajola, 5. Corte, 6. Aléria, A. Corse du deçà des Monts, 7. Ajaccio, 8. Vico, 9. Sartène, 10. Bonifacio, B. Corse du delà des Monts.

11 Il n'y a donc aucune commune mesure entre ces pourcentages et les chiffres retenus jusqu'à présent. Il n'en demeure pas moins vrai que la Corse, au début du XVIII ${ }^{\mathrm{e}}$ siècle, 
se trouve affectée d'un taux d'homicide supérieur, en moyenne, à ceux qui ont été calculés pour de nombreuses villes européennes au Moyen Âge ${ }^{25}$. En conséquence, on est loin, en ce domaine précis, des réalités européennes du temps. Loin, par exemple du Lyonnais et du Beaujolais étudiés par Françoise Bayard, où, dans un espace qui englobe la deuxième ville de France et compte plus de 600000 habitants à la veille de la Révolution, l'on ne dénombre que 263 meurtres entre 1604 et $1790^{26}$.

\section{La géographie de la violence homicide}

Une approche régionale ne laisse pas apparaitre de contrastes substantiels entre les deux grandes subdivisions administratives. La Corse du deçà des Monts, qui correspond à l'actuel département de la Haute-Corse, bien qu'enregistrant 583 meurtres, c'est-àdire $61,6 \%$ de l'ensemble pour la période 1702-1720, est vraisemblablement moins criminogène que la Corse du delà des Monts dont la population est quelque deux fois et demie moins importante.

13 Le passage à la circonscription administrative de base qu'est la province ${ }^{27}$ permet d'affiner cette première analyse. On peut, de prime abord, souligner l'impact des "grandes» villes insulaires que sont Bastia et $\mathrm{Ajaccio}^{28}$. Mais toutes deux coiffent sur le plan administratif de vastes zones rurales qui comptent, du moins pour ce qui concerne la province de Bastia, parmi les plus peuplées de l'île; et la part de la criminalité de sang spécifiquement urbaine se révèle vraiment marginale. En effet, abstraction faite de la période 1702-1704 pour laquelle nous ne disposons que d'un décompte global des morts violentes, la ville de Bastia est créditée de 22 meurtres sur un total de 239 attribué à la province et le rapport est de 16 pour 192 homicides pour la ville d'Ajaccio, soit respectivement 9,2 et $8,3 \%$. Retenons surtout que ces deux provinces affichent 501 homicides, ou $52,9 \%$ du total pour la période considérée. L'étude comparative de la situation de ces deux entités spatiales et administratives est encore plus signifiante. Elle met en exergue la dangerosité de la province d'Ajaccio où sont perpétrés $65,8 \%$ des meurtres enregistrés dans le delà des Monts (239 sur 363) alors que la province de Bastia avec $44,8 \%$ des crimes de sang commis dans le deçà des Monts semble, si l'on peut dire, relativement épargnée.

14 À quoi tiennent ces disparités ? Elles semblent résulter de la conjonction de plusieurs facteurs. En sus d'une meilleure couverture policière due au fait que Bastia est le lieu de résidence du gouverneur et la capitale administrative de l'île, il faut également prendre en compte des réalités sociologiques très différentes qui sont accentuées à cette époque par un inégal développement économique.

15 La province d'Ajaccio correspond en partie à l'ancienne Terre dite des seigneurs, région d'où la féodalité n'a été extirpée, et encore incomplètement, qu'au début du XVII siècle. Si les anciens féodaux ont perdu nombre de leurs privilèges, ils ont par contre conservé beaucoup de leur morgue passée et surtout la quasi-totalité de leurs terres. Ils ont continué à les faire exploiter sur un mode traditionnel par les descendants de leurs anciens vassaux qui, bien que constituant une main-d'œuvre relativement nombreuse, et par là même bon marché, n'en sont pas pour autant très dociles, à l'image de leurs ancêtres qui ont massacré leurs maîtres en $1615^{29}$. Or, dans cette région, la très grande partie de la population vit toujours majoritairement ou partiellement de l'élevage. La politique de développement agricole initiée par Gênes à partir des années $1637^{30}$, parce qu'elle s'accompagne d'enclosures et de passe-droits au bénéfice des notables, va 
provoquer rapidement de graves tensions au sein d'une société très inégalitaire qui n'a rien oublié de ses anciennes dissensions et où chacun cultive un sens fort aigu de l'honneur. Ces conditions générales et cette hiérarchie spécifique des valeurs perdurent à l'époque qui nous intéresse. Elles génèrent une violence latente qui, fréquemment, trouve un exutoire dans le meurtre.

L'univers économique et mental des gens de la province de Bastia a quelque peu évolué. Nous sommes ici dans la partie la plus prospère d'un vaste espace qui, en gros, englobe la Haute-Corse actuelle, à l'exception du Cap Corse, et que l'on nomme la Terre du commun depuis que les féodaux en ont été définitivement chassés au XIV ${ }^{e}$ siècle, grâce au soutien de la commune de Gênes. De ce côté-ci des Monts, l'emprise de la Dominante est plus affirmée et elle se renforce à mesure que l'on se rapproche du centre du pouvoir, à savoir Bastia. En outre la province englobe des microrégions qui comptent parmi les plus riches de l'île et qui ont vu leurs potentialités naturelles heureusement stimulées par la politique génoise ${ }^{31}$. L'affirmation de la propriété privée et par là même l'extension des enclosures ont bien sûr multiplié, ici aussi ${ }^{32}$, les sujets de tension. Mais les habitants de la Castagniccia, du Nebbio et de la Casinca travaillent maintenant en relation étroite avec le marché et le port bastiais. Ils apparaissent plus policés que leurs homologues du Sud, plus proches du monde génois. D'ailleurs, à ce stade, il est intéressant de mettre en relation leur comportement avec celui des Cap Corsins qui, depuis longtemps, contrôlent les circuits commerciaux unissant l'île et la métropole et dont la province (Rogliano) est de loin la moins criminogène de l'île.

Mais, finalement, tout ceci est affaire de nuances plutôt que de tendances bien marquées. Si l'impact de cette criminalité de sang, indépendamment des densités de population, peut varier sensiblement selon les espaces considérés en fonction de facteurs sociologiques, économiques ou historiques divers, elle revêt avant tout un caractère multiforme. En dehors des périodes de troubles généralisés qui, comme ce sera le cas à partir des années 1729-1730, voient se constituer de véritables bandes organisées qui mettent en coupe réglée des régions entières, elle est bien la résultante d'innombrables dérives individuelles et, telle une chape de plomb, elle pèse uniformément sur toutes les provinces.

Ainsi, si l'on se réfère à la typologie, retenue par les gouverneurs de la Corse, qui distingue les meurtres intentionnels, découlant ou non d'une vendetta et les homicides survenus lors d'une rixe, on ne voit apparaître aucune disparité régionale. Partout cela semble tenir aux circonstances tout autant qu'aux valeurs véhiculées par la société corse qui, à l'instar de bien d'autres régions d'Europe à cette époque, place au premier rang non seulement la défense de l'honneur individuel ou familial mais aussi, et de plus en plus, celle de la propriété.

\section{La nature des homicides}

Des données présentées dans le tableau $\mathrm{n}^{\circ} 2$, il ressort en première lecture que les circonstances de 397 meurtres (35,3\%) ne sont pas élucidées ou, en tout cas, qu'aucun détail n'est mentionné qui nous permettrait de nous forger une opinion à ce sujet. Cependant, l'identité des meurtriers est presque toujours connue. Cela est déjà éclairant quant aux facultés d'investigation de la police génoise, pourtant si décriée, qui s'efforce toujours de distinguer les homicides commis intentionnellement, dans le cadre ou non d'une vendetta, de ceux qui résultent de rixes. En définitive il apparaît 
que, à moyens d'investigation constants, les résultats obtenus tiennent au plus ou moins grand investissement des forces de police et de justice et à l'ardeur dont font preuve les Giusdicenti, le gouverneur en tête, pour les motiver ${ }^{33}$. En fait, si l'on fait abstraction des enquêtes menées durant les années 1710-1714 et durant le biennio 1692-1694 pour lequel les informations sont lacunaires et ne concernent que la seule province de Bastia, on se rend compte qu'au contraire les circonstances de la grande majorité des meurtres ou des morts violentes sont élucidées et permettent une ventilation relativement fine selon leur nature et leur impact respectif: meurtres intentionnels, homicides survenus lors de rixes, morts accidentelles, éliminations de bandits (ou de bannis).

Tableau $n^{\circ} 2$ - Les circonstances des homicides

\begin{tabular}{|c|c|c|c|c|c|c|}
\hline PERIODES & \multicolumn{5}{|c|}{ CIRCONSTANCES DU MEURTRE } & \multirow[t]{2}{*}{ ENSEMBLF } \\
\hline & $\begin{array}{c}\text { MEURTRES } \\
\text { INTENTIONNELS }\end{array}$ & RIXES & $\begin{array}{c}\text { MORTS } \\
\text { ACCIDENTELLES }\end{array}$ & $\begin{array}{l}\text { BANDITS } \\
\text { ÉLIMINÉS }\end{array}$ & $\begin{array}{l}\text { CIRCONSTANCES } \\
\text { INDÉTERMINÉES }\end{array}$ & \\
\hline $1690-1692$ & 37 & 18 & 4 & 4 & 47 & 110 \\
\hline $1692-1694$ & & & & & 67 & 67 \\
\hline $1694-1702$ & & & & & & \\
\hline $1702-1704$ & 43 & 76 & - & 20 & - & 139 \\
\hline 1704-1706 & 65 & 90 & 45 & 23 & - & 223 \\
\hline $1706-1708$ & 43 & 37 & 2 & - & 3 & 85 \\
\hline $1708-1710$ & & & & & & \\
\hline $1710-1712$ & 27 & 23 & 1 & - & 95 & 146 \\
\hline $1712-1714$ & 4 & 6 & 1 & 5 & 124 & 140 \\
\hline $1714-1716$ & 54 & 27 & 18 & 5 & 26 & 130 \\
\hline $1716-1718$ & 10 & 13 & 2 & 4 & 24 & 53 \\
\hline $1718-1720$ & 7 & - & 9 & - & 14 & 30 \\
\hline $1690-1720$ & 290 & 290 & 82 & 61 & 400 & 1123 \\
\hline
\end{tabular}

Les deux premières catégories s'équilibrent et regroupent le plus grand nombre de décès et, si l'on en juge d'après ces données, la très grande majorité des meurtres dont on ignore les circonstances sont certainement à reporter assez équitablement dans ces deux rubriques. Viennent ensuite les morts violentes qui sont assimilées à des homicides involontaires ou accidentels. 
21 En vérité, sous cette appellation, se cachent des réalités très diverses. La majorité des cas concerne des accidents champêtres. Ainsi dans les années $1704-1706^{34}$, période durant laquelle on enregistre le plus grand nombre de décès accidentels, entre autres cas, on relève que dans, la province d'Aléria, Simon Paolo du feu Orsilio a été tué, le 18 octobre 1704 par le retour du moyeu de la meule du moulin où il travaillait. Gio Battista du feu Orso Paolo de Lugo di Nazza s'est noyé le 28 février 1705 et la même mésaventure est arrivée à Orso Paolo de Zallana le 22 mars de la même année. Le 25 mai 1705, Domenico Sarzana meurt sous les sabots d'un cheval à Migliacciaro. Maria Domenica, fille de Mar'Antone du Muchietto, se tue en tombant d'un cerisier le 21 juin 1705. Maria Francesca, fille de Pasquino, a été tuée par un sanglier le 9 octobre 1705. Pour la Balagne, durant le même biennio, est signalée, sous le n²54, la mort de Gio Zucca de Pier Matteo survenue à Aregno des suites d'une blessure occasionnée par le tir de sa propre arquebuse... Sous le $n^{\circ} 268$, on note le décès de deux enfants tombés du toit, vraisemblablement en terrasse, d'une maison de Novella. À Arbitro de Bozio, dans la province de Corte, toujours sous la rubrique des décès accidentels, Donna Antonia est, sans autre précision, dite morta da sé, ce qui signifie qu'elle s'est suicidée.

À Calvi, en 1716, Simon Gio d'Angelo Martino est dit tué accidentellement d'un coup de marcoletto par un autre enfant. Enfin, dans la province de Vico, après un décès occasionné par un coup de pied de cheval, est mentionné, en date du 17 juin 1715, le meurtre de Giovanni, fils de Giovanello de Vico, âgé seulement de neuf ans, survenu, d'après l'enquête, plutôt par accident que pour tout autre raison ${ }^{35}$.

On pourrait multiplier les exemples. Tous prouvent que la plus grande partie des morts violentes, dites accidentelles, est bien due aux conditions très pénibles qui régissent la vie et les activités du monde rural, mais il n'en demeure pas moins vrai que certains décès regroupés sous cette rubrique seraient de nos jours qualifiés autrement.

La violence en effet est partout, et, même en l'absence d'un contexte hostile, une brutalité latente peut, aux marges, faire des victimes. Cependant, elle explose et provoque des ravages essentiellement dans des situations conflictuelles, dans le contexte de vieilles rancunes inter-familiales souvent pieusement entretenues sur plusieurs générations, ou bien à l'occasion de disputes entre individus au sang chaud, ou encore entre groupes dont les membres sont liés par des intérêts divers. Toujours, avec ou sans préméditation, le moindre dérapage verbal peut provoquer l'irréparable.

Bien sûr, en Corse aussi, la préméditation est toujours considérée comme un fait aggravant et ce d'autant plus qu'elle est, dans l'île, presque toujours synonyme de vengeance.

Toutefois, à l'examen d'informations - généralement laconiques - livrées par les autorités judiciaires, on ne peut se faire une opinion définitive quant à l'impact de la vendetta sur la criminalité dite intentionnelle. Mais, quelles que soient les motivations ou les circonstances réelles du meurtre, il semble que les autorités génoises y voient toujours, et avec raison, une relation étroite de cause à effet avec ce phénomène. En effet, ici, il s'est avéré depuis longtemps que tout meurtre, intentionnel ou non, qui n'est pas rapidement sanctionné par un jugement et surtout par une condamnation effective donne naissance à une dérive vindicative qui implique les membres de la famille de la victime et ceux de la famille de l'assassin et se traduit à moyen terme en un véritable bain de sang. 
Ceci étant posé, le propos de cette étude n'est pas de quantifier les ravages de la vendetta en Corse $^{36}$ mais plutôt de cerner au plus près, à partir d'exemples précis, la réalité d'une criminalité de sang qui, bien qu'étant beaucoup plus faible qu'on ne l'imaginait jusqu'à présent, n'en pèse pas moins lourdement sur la société insulaire et influe insidieusement sur le comportement de chacun.

Si les renseignements fournis par les documents en notre possession ne nous permettent qu'occasionnellement de suivre les affaires jusqu'à leur terme, c'est-à-dire jusqu'à la sanction, ils révèlent cependant que les meurtres, qu'ils soient catalogués comme étant intentionnels, ou bien comme étant survenus lors d'une rixe, font l'objet d'une attention particulière, ce qui nous permet de mieux appréhender au quotidien cette violence sanguinaire. À partir de l'année 1704, la date du meurtre commence à être indiquée, et jusqu'en 1720 l'identification du mois est assurée dans $62,4 \%$ des cas $^{37}$.

Tableau $n^{\circ} 3$ - Les temps de la violence

\begin{tabular}{|l|l|l|l|l|l|l|l|l|l|l|l|l|l|}
\hline 1704-1720 & Janv. & Fév. & Mars & Avril & Mai & Juin & Juil. & Août & Sept. & Oct. & Nov. & Déc. & Total \\
\hline Nbre & 35 & 31 & 44 & 42 & 37 & 56 & 59 & 41 & 50 & 36 & 32 & 41 & 504 \\
\hline$\%$ & 7 & 6,2 & 8,7 & 8,3 & 7,3 & 11,1 & 11,7 & 8,1 & 9,9 & 7,1 & 6,4 & 8,1 & 100,00 \\
\hline
\end{tabular}

Ces données ne révèlent pas de grands contrastes. On peut être tué en Corse en toutes saisons. Cependant le caractère criminogène de la période estivale est bien affirmé, 41, $3 \%$ des meurtres datés sont effectivement commis entre juin et septembre, avec une pointe marquée pour le dernier mois du printemps et le premier mois de l'été. À peu de chose près, on enregistre pour les mois de juin et juillet les mêmes pourcentages que ceux qui sont présentés par Robert Muchembled ${ }^{38}$, pour l'Artois des $\mathrm{XV}^{\mathrm{e}}-\mathrm{XVII}{ }^{\mathrm{e}}$ siècles, à partir des lettres de rémission qui, il est vrai, recouvrent une réalité homicide particulière.

La violence de saison chaude, en Corse comme en Artois, n'exclut pas totalement des causes hormonales, ici aussi les travaux des champs et surtout les moissons sont favorables aux rencontres amoureuses; et combien de sourires à peine esquissés, mais interceptés et interprétés par des rivaux particulièrement ombrageux, furent-ils à l'origine de querelles qui se terminèrent tragiquement? Mais surtout, la belle saison est l'époque où l'on peut le plus facilement guetter son ennemi et l'abattre d'un coup d'arquebuse au détour d'un sentier ou dans son champ.

31 Paradoxalement il s'avère que l'arquebuse à rouet est aussi l'arme la plus communément utilisée dans les rixes alors que dans ce genre de rencontre au corps à corps, on aurait plutôt imaginé que l'on utilisât des armes blanches.

Tableau $n^{\circ} 4$ - Les armes du meurtre

\begin{tabular}{|l|l|l|l|l|l|l|l|l|l|}
\hline PÉRIODES & \multicolumn{7}{|c|}{ NATURE DE L'ARME } \\
\hline & $\begin{array}{l}\text { Arme } \\
\text { à feu }\end{array}$ & $\begin{array}{l}\text { Arme } \\
\text { blanche }\end{array}$ & Bâton & $\begin{array}{l}\text { Coups } \\
\text { de pied }\end{array}$ & Pierres & $\begin{array}{l}\text { Accident } \\
(1)\end{array}$ & Autres & $\begin{array}{l}\text { Sans } \\
\text { précision }\end{array}$ & Ensemble \\
\hline
\end{tabular}




\begin{tabular}{|l|l|l|l|l|l|l|l|l|l|}
\hline $1690-1692$ & & & & & & & & 110 & 110 \\
\hline $1692-1694$ & & & & & & & & 67 & 67 \\
\hline $1694-1702$ & & & & & & & & & \\
\hline $1702-1704$ & & & & & & & & 139 & 139 \\
\hline $1704-1706$ & 34 & 3 & - & 1 & - & 45 & 2 & 138 & 223 \\
\hline $1706-1708$ & 55 & 6 & - & - & 1 & - & - & 23 & 85 \\
\hline $1708-1710$ & & & & & & & & & \\
\hline $1710-1712$ & 47 & 5 & 2 & 1 & 1 & 1 & - & 89 & 146 \\
\hline $1712-1714$ & 13 & - & - & - & 1 & - & 2 & 124 & 140 \\
\hline $1714-1716$ & 37 & 13 & - & 1 & 1 & 6 & 3 & 69 & 130 \\
\hline $1716-1718$ & 6 & 20 & 3 & - & 10 & 2 & - & 12 & 53 \\
\hline $1718-1720$ & 12 & 6 & 1 & - & - & 8 & - & 3 & 30 \\
\hline $1690-1720$ & 204 & 53 & 6 & 3 & 14 & 62 & 7 & 774 & 1123 \\
\hline $1704-1720$ & 204 & 53 & 6 & 3 & 14 & 62 & 7 & 458 & 807 \\
\hline
\end{tabular}

(1) Accidents dont les circonstances sont connues.

32 Le tableau $\mathrm{n}^{\circ} 4$ qui répartit les meurtres en fonction de l'arme utilisée, met parfaitement en évidence l'impact de l'arquebuse à rouet, et cela même si les relations de ces meurtres ne mentionnent pas toujours la nature de l'arme utilisée. En effet, cette information n'apparaît dans les états qu'à partir de 1704 et encore de manière très irrégulière. Pour l'ensemble de la période, abstraction faite des décès accidentels, elle ne figure que 287 fois pour 1062 meurtres, c'est-à-dire dans seulement $27,02 \%$ des cas. Cependant si, en toute logique, l'on ne retient que la période 1704-1720, cette proportion grimpe à $38,47 \%$ et confère une crédibilité incontestable à nos données. Il en ressort qu'en Corse, aux Temps Modernes, dans $71 \%$ des cas, c'est-à-dire presque trois fois sur quatre, la mort était donnée au moyen d'une arme à feu. Loin derrière, venaient les armes blanches $(19,16 \%)$, et à défaut d'armes on n'hésitait pas à utiliser tout ce qui pouvait en faire fonction, les pierres $(4,8 \%)$, le bâton $(2 \%)$, les coups de pieds $(1 \%)$ ou encore tout instrument se trouvant à portée de main de l'assassin potentiel $(2,4 \%)$.

\section{La violence au quotidien}

33 C'est donc généralement avec une arquebuse que l'on assouvit une vengeance et que l'on abat quelqu'un avec qui l'on a eu un différend parfois longuement ressassé. Dans les deux cas de figure, bien que la cause soit rarement explicitée, il est toujours 
mentionné que le meurtre est intentionnel. Dans la province de Vico, le 5 avril 1706, Matteo fils du feu Antonetto de Lenno est tué intentionnellement d'un coup d'arquebuse par Anton Francesco de feu Giulio de Vico ${ }^{39}$. La victime et le meurtrier sont dits exempts jusqu'alors de toute condamnation. Le même jour, Antonio, originaire de Vico, est abattu lors d'une rencontre, toujours par un tir d'arquebuse, par Carlo fils du feu Gio du feu San Simone de Balogna, un criminel condamné au bannissement, au prétexte que c'était son ennemi ${ }^{40}$. Dernier cas parmi tant d'autres que l'on pourrait mettre en exergue, c'est encore au moyen d'une arquebuse qu'Anton Giacinto du feu Bastiano de Nocario d'Orezza est abattu délibérément, le 12 décembre 1714, par Gio Alfonso fils de Giovacchino de Scata d'Ampugnani ${ }^{41}$. Si les homicides intentionnels par arme à feu sont très majoritairement le fait d'une seule personne, il arrive cependant aussi que la victime périsse sous le feu croisé de plusieurs assassins qui lui ont tendu un guet-apens. C'est ainsi que dans la province de Sartène, André fils d'Orsone de Fozzano tombe, le 7 mai 1705, sous les balles de huit hommes, clairement identifiés, du village d'Albillare ${ }^{42}$. Mais, c'est aussi par ce même moyen que l'on règle souvent des querelles nées dans l'instant, impliquant généralement, comme nous l'avons déjà souligné, l'affrontement corps à corps, et que l'autorité judiciaire qualifie de rixes. Francesco Peri d'Ajaccio fut ainsi tué involontairement d'un coup d'arquebuse dans une rixe survenue le 8 mars $1707^{43}$. Le 5 avril de la même année, Antonio du feu Gio Francesco de la Prugna, province d'Ajaccio fut mortellement blessé d'un tir d'arquebuse, dans une rixe ${ }^{44}$. Le 4 novembre 1714, c'est toujours par des décharges d'arquebuses, que Giulio Francesco et Stefano, fils et frère du feu Paolo Francesco de Rapallo du Nebbio, furent abattus, lors d'une rixe, par Giulio Francesco et ses comparses, tous habitants de ce dit lieu ${ }^{45}$.

Dans l'esprit des greffiers qui consignent ces faits il ne semble pas y avoir de confusion entre ces crimes commis sous le coup de la colère et dans le cadre d'un affrontement aussi soudain que brutal et des assassinats prémédités, suivant d'assez près une éventuelle querelle. En effet, ce dernier schéma est aussi retenu comme, par exemple, le 28 août 1707 à Calenzana, gros bourg de la juridiction de Calvi où, le 28 août 1707, Angelo Maria de feu Santo fut abattu au moyen d'une arquebuse par Giovanni du feu Anto Gio dudit lieu à cause d'une rixe survenue quelques heures auparavant dans ce bourg.

35 L'arquebuse à rouet avait été introduite en Corse lors de la Guerre dite des Français, et les Corses fascinés par ses possibilités tant offensives que défensives excellèrent très rapidement dans son maniement. Dès lors, la paix revenue, l'autorisation de porter cette arme, ainsi que des armes blanches, devinrent des moyens forts attractifs dont Gênes sut se doter pour inciter les insulaires à répondre à ses sollicitations. Non seulement la licence de port d'arme et en particulier de l'arquebuse récompensera assez tôt ceux qui s'étaient distingués à son service lors des dernières guerres, mais Gênes utilisa aussi ce moyen à des fins économiques et plus particulièrement, à partir des années 1737-1738, pour inciter les notables corses à participer massivement à son entreprise de mise en valeur agricole de l'île ${ }^{46}$. Malheureusement, l'obtention d'une licence de port d'armes, si recherchée et réservée - dans un premier temps - à une minorité privilégiée et méritante, ne fut bientôt plus qu'un instrument fiscal au service d'un État avare de ses deniers, et de ses serviteurs trop souvent peu scrupuleux. C'est ainsi que les licences ou patentes de port d'arme se multiplièrent dans la deuxième moitié du XVII ${ }^{e}$ siècle et c'est pourquoi il nous faut imaginer, à cette époque, une 
multitude d'individus se déplaçant, en toute impunité, bardés non seulement d'armes blanches mais porteurs aussi d'armes à feu et prêts à les utiliser à la moindre provocation.

Nous sommes bien là au cœur des préoccupations de l'administration génoise qui, avec raison mais bien tardivement, prend conscience que la prolifération des armes à feu et plus particulièrement de l'arquebuse à rouet est devenue un vecteur particulièrement aggravant de la criminalité de sang. Dès 1711, les représentants des populations du deçà des Monts, les Nobles Douze, en avaient dénoncé les effets pervers et demandé la suppression des patentes. La République, après bien des hésitations, s'engagera dans cette voie en 1715 et, bien que tous ceux qui se sont intéressés à cette période, aient contesté l'efficacité de cette décision, au motif que son application fut très vite pervertie, ce qui est exact, celle-ci - c'est indéniable aussi - va avoir, du moins dans l'immédiat, des effets bénéfiques. Dès 1716, les meurtres chutent de façon très significative. On passe d'une moyenne de 139 homicides par biennio pour la période allant de mai 1710 à mai 1716 à seulement 53 dans les années 1616-1618, soit une diminution de près de $60 \%$ !

Bien entendu, il faut également tenir compte des conditions de vie du temps. Les valeurs qu'elles génèrent, nous l'avons déjà souligné, poussent au premier plan des préoccupations de la multitude, non point le respect de la vie, mais la préservation de l'honneur et la défense de la propriété. Aussi, outre cette arme très performante, tous les moyens semblent bons aux contemporains pour assouvir leur haine de l'autre ou pour s'imposer. Dans ce contexte, la montée spectaculaire, dès la fin de l'année 1715, des crimes commis au moyen d'une arme blanche, d'un bâton ou de pierres doit retenir notre attention. C'est ainsi que les Corses suppléent l'abandon, tout à fait provisoire, des armes à feu.

En fait, bien que n'étant pas spécifique à la Corse, loin s'en fallait, la brutalité était une donnée incontournable de la société insulaire de ce temps et elle se traduisait souvent par des dérives extrêmement cruelles dont étaient victimes non seulement les hommes, mais aussi les femmes et les enfants. Le 20 janvier 1715 à Tomino dans le Cap Corse, région qui n'était pas la moins policée de l'île, la jeune Antonia fille du feu Domenico fut tuée au moyen d'une broche, par sa mère, Imperatrice, qui, selon ses dires, utilisa cet instrument pour la corriger. Imperatrice s'en tirera avec une condamnation par contumace à trois ans de relégation sur l'île voisine de Capraia ${ }^{47}$. L'autorité parentale apparaît donc sans limites et elle est confortée par la législation. Cependant, les femmes semblent encore plus exposées que les enfants à cette brutalité latente ${ }^{48}$. Pour la période étudiée, trente-six femmes furent assassinées, dont douze des mains de leurs époux qui employèrent pour ce faire les moyens les plus divers, les coups de pieds (trois cas), l'arquebuse (deux), le poignard (deux) et le lacet (un). Mais la violence est généralisée, les femmes prennent parfois les devants et se débarrassent de leur mari. Ainsi le 15 août 1714, Francesca Maria de Bastelica empoisonne son époux Gio Angelo, avec la complicité d'un certain Ventura, qui, vraisemblablement, est son amant ${ }^{49}$. Par ailleurs, elles n'hésitent pas à participer à des rixes, où certaines d'entre elles succombent, parfois dans des conditions atroces, comme ce fut le cas de Diana, épouse de Domenico Bozio de Bastia, massacrée à coups d'entrave métallique, le $1^{\mathrm{er}}$ septembre 1710, par le notable Carlo Giuseppe Bonavita ${ }^{50}$. D'autres, par contre, l'emportent sur leur adversaire du sexe fort, telle Contessa, épouse de Francesco de Corbara en Balagne, 
qui le 10 août 1711 tue à coups de pierres Santo du feu Giacomo, originaire du même lieu $^{51}$.

La défense de la propriété peut conduire aussi aux pires excès, auxquels succombent même des ecclésiastiques ${ }^{52}$, à l'instar du prêtre Domenico Acciebelli de Prunelli di Migliacciaro qui, le 4 novembre 1715, abattit d'un coup d'arquebuse Martino du feu Giovanni, soldat appartenant à la compagnie des chevaux-légers d'Aléria, lors d'une rixe survenue à la suite d'un différend concernant des terrains. Et que dire du comportement de Pietro, fils du feu Pier Battista, de Castiglione de Giovellina dans la province de Corte, qui surprenant, le 30 octobre 1717, Agata épouse de Solidoro, dudit lieu, une femme enceinte, en train de cueillir des glands sur un de ses chênes verts, la frappa sauvagement à coups de pieds et de pierres au point de provoquer l'avortement et de la tuer ${ }^{53}$. Ce crime, hors du commun, fut lourdement sanctionné et son auteur condamné aux galères à vie. Mais une fois encore par contumace !

\section{En l'absence de moyens}

Le jugement par contumace était en effet devenu un expédient utilisé de manière quasi systématique par des autorités judiciaires incapables de se saisir de coupables qui, aussitôt leur délit commis, se réfugiaient dans le maquis où ils pouvaient tenir pendant des mois, et bien plus longtemps encore, avec l'aide et la complicité de leurs familles. Ces pratiques discréditaient la justice et entretenaient dans l'île un climat d'insatisfaction et d'insécurité car ces manifestations de violence exacerbée étaient d'autant plus traumatisantes pour la population que la plupart du temps elles demeuraient impunies, alors que, sans doute par réaction, des délits mineurs étaient souvent sévèrement réprimés. Nous touchons là du doigt la cause structurelle de l'inefficacité de la justice génoise qui, parce qu'elle était dans l'impossibilité de se saisir tant des auteurs d'homicides que de criminels de moindre importance et de les sanctionner, a été perçue, à juste titre, comme un des principaux agents de l'extension de la vendetta.

Pourtant, bien que le but de cette enquête ne soit pas de réécrire l'histoire du système judiciaire génois, nous nous devons de dire que ce jugement aussi mérite d'être nuancé. Avec la réactualisation des Statuts civils et criminels de la Corse, publiés en $1571^{54}$, et en adaptant constamment la législation en fonction des besoins, Gênes avait doté l'île d'un corpus de lois ${ }^{55}$ qui, en matière criminelle, semblait à la hauteur des enjeux. Il prévoyait même, dans un souci d'efficacité qui offusquait la morale sinon le droit, d'impliquer les insulaires dans la chasse aux bandits en instaurant un système de récompenses qui légalisait non seulement des primes pécuniaires, mais aussi des échanges, sanction contre sanction, tête contre tête. Ce qui permettait d'obtenir la grâce d'un tiers contre la capture ou l'élimination physique d'un bandit condamné à la même peine ${ }^{56}$. C'est ainsi que furent abattus la plupart des bandits dont nous avons enregistré le décès. Mais nous devons insister notamment sur le fait que les enquêtes étaient menées très correctement, vraisemblablement avec le concours des familles des victimes ${ }^{57}$ et à coup sûr avec celui des autorités communales ${ }^{58}$, et il est intéressant à noter que le coupable est identifié dans la quasi-totalité des affaires. C'est du moins l'enseignement qui se dégage de l'examen des relations qui prennent en compte cette étape décisive de la procédure, et qui mentionnent par trois fois seulement qu'une affaire n'est point élucidée. 
Tableau $n^{\circ} 5$ - Les effectifs du (ou des) barigel (s) de Corse

\begin{tabular}{|l|l|l|l|}
\hline Provinces & Début XVII & Milieu XVII & $1726 / 1728$ \\
\hline Bastia & 23 & 44 & 72 \\
\hline Rogliano & & & 7 \\
\hline Calvi & & & 11 \\
\hline Corte & 7 & 10 & 12 \\
\hline Ajaccio & 10 & 17 & 26 \\
\hline Vico & & & 13 \\
\hline Sartène & 4 & 10 & 10 \\
\hline Bonifacio & & 8 & 8 \\
\hline Ensemble & $\mathbf{4 4}$ & $\mathbf{8 9}$ & $\mathbf{1 5 9}$ \\
\hline
\end{tabular}

42 En définitive, c'est surtout le bras de la justice qui est en cause. Quasiment toutes les affaires que nous avons étudiées et qui ont abouti à un jugement définitif en témoignent: le coupable, une fois identifié, faute d'être appréhendé, était jugé par contumace et entraîné de ce fait à venir grossir les rangs des brigands qui infestaient les campagnes. Donc, ce dysfonctionnement tient essentiellement à la faiblesse des effectifs policiers engagés sur le terrain par Gênes. Tous ceux qui jusqu'à présent se sont intéressés à cette problématique, ont souligné ce fait, sans toutefois être en mesure d'avancer des estimations satisfaisantes quant à l'importance réelle des forces de police. Celles-ci, à l'époque qui nous intéresse, et depuis longtemps déjà, étaient centrées sur les chefs-lieux des provinces dans le cadre d'une structure appelée le bargello, qui était l'équivalent italien du barigel français. Le premier barigel sera installé à Bastia où l'on distinguera rapidement un bargello di città, chargé du maintien de l'ordre dans la ville, et un bargello di campagna, plus particulièrement chargé de contrôler l'arrière-pays. Puis, cette organisation sera étendue aux autres provinces, au fur et à mesure de leur création ou de leur affirmation, selon une progression dont le tableau $n^{\circ} 5$ rend partiellement compte.

Nous n'avons, il est vrai, aucun renseignement sur l'importance du bargello d'Aléria, territoire qui initialement dépendait de la juridiction de Corte et où un lieutenant ne sera installé qu'en 1640, ni sur celui d'Algajola qui était vraisemblablement moins important car pour l'essentiel les forces de maintien de l'ordre de la Balagne étaient

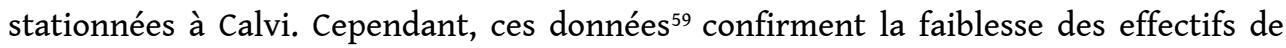
police dans l'île. Pour la période qui nous intéresse, ils s'élevaient à 159 hommes ou «famegli $»^{60}$ pour huit provinces et dans la meilleure des hypothèses à 180 pour l'ensemble de la Corse, ce qui était tragiquement insuffisant eu égard à la situation que nous avons évoquée. 
D'où la pratique pernicieuse de l'indulto, pardon général ou spécifique, qui permettait de purger régulièrement les campagnes de leur trop-plein de bannis et de bandits, et de diriger ceux-ci, munis de sauf-conduits délivrés par le gouverneur, vers les armées génoises, ou vers d'autres contées du continent ${ }^{61}$. Passé quelque temps, ils pouvaient revenir en toute impunité dans l'île, après avoir obtenu le pardon de leurs adversaires. Et ainsi le cycle de la violence était constamment entretenu. D'où aussi, en dernière extrémité, la suppression en 1715 des patentes de port d'armes à feu, et la tentation pour la Sérénissime République fort consciente de ces multiples déficiences, mais qui n'entendait pas pour autant consentir un effort financier supplémentaire, d'impliquer encore davantage, au risque d'intensifier la vendetta, les insulaires dans la chasse aux bandits, partant de l'adage ${ }^{62}$ qui voulait que seul un Corse était capable de capturer un autre Corse.

\section{BIBLIOGRAPHIE}

Acinelli, F. M., Storia veridica della Corsica, manuscrit, Gênes, vers 1770, transcription réalisée par l'A.D.E.C.E.C. à Cervione et l'Association Franciscorsa, Bastia, 1994.

Bayard, F., Les crimes de sang en Lyonnais et en Beaujolais aux XVII ${ }^{\mathrm{e}}$ et XVIII siècles, in Garnot, B. (Dir.), Histoire de la criminalité de l'Antiquité au XXe siècle, Publication de l'Université de Bourgogne, LXXI, Série du Centre d'Études Historiques - I -, 1972, pp. 273-281.

Borlandi, F., Per la storia della popolazione della Corsica, Gênes, 1942.

Busquet, J., Le droit de la vendetta et les paci corses, $2^{\mathrm{e}}$ édition, Joué-Lès-Tours, La Simare Éditions, CTHS, 1994.

Caraffa, M.V., de (Ed.), Mémoires historiques sur la Corse par un officier du régiment de Picardie; 1744-1777, inBulletin de la Société des Sciences historiques et Naturelles de la Corse (BSSHN), Bastia, 1889.

Caraffa, Ph., de, (Ed.), Visita della diocesi di Mariana ed Accia fatta nell'anno 1740 per deputazione dell''llustrissimo et Reverendissimo Monsignor Agostino Saluzzo, vescovo, BSSHN de la Corse, mai-juin,1890, pp.131-238.

Coppolani, J.-Y., Serpentini, A. L., (présentés et traduits par) Les statuts civils et criminels de la Corse, Ajaccio, Éditions Albiana, 1998.

Dupâquier, J, Histoire de la population française, Paris, PUF, 1988, T. 2.

Garnot, B., L'historiographie de la criminalité pour la période moderne, in Garnot, B., (Dir.), Histoire de la criminalité de l'Antiquité au XX $X^{e}$ siècle; nouvelles approches, Publication de l'Université de Bourgogne, LXXI, Série du Centre d'Études Historiques - I -, 1992, pp. 25-29.

Gaudin Abbé,Voyage en Corse et vues politiques, Paris, Lefevre Libraire, 1787.

Ghiara, C., Le fonti criminali genovesi: sondaggi seriali o culturali? Quaderni Storici, 1979, 44.

Graziani, A.-M., Stromboni, J., Les feux de la Saint-Laurent, Ajaccio, Éditions Alain Piazzola, 2000. 
Gregorj, G. C., Statuti civili e criminali di Corsica, Lyon, Imprimerie du Moulin, Ronet et Sibuet, 1843. Le Roy Ladurie, E., Histoire de la France rurale, Paris, Éditions du Seuil, 1975.

Muchembed, R., La violence au village. Sociabilité et comportements populaires en Artois du XV au XVII ${ }^{\mathrm{e}}$ siècles, Éditions Brepols, Turnhout, 1989.

Natali, G. M., Disinguano intorno alla Guerra di Corsica scoperto da Curzio Tulliano Corso ad un suo amico dimorante nell'Isola, Livourne, 1736, Édition, traduction, notes et commentaires d'Arrighi J.-M. et Castellain, Ph., Ajaccio, Librairie La Marge, 1983.

Pomponi, F., Banditisme corse et ordre génois in Coll., Banditisme et violence sociale dans les sociétés de l'Europe méditerranéenne, Études corses, Ajaccio, La Marge Édition, 1995, pp. 291-323.

Raggio, O., Faide e parentele, lo Stato genovese visto da Fontanabruna, Microstorie 18, Torino, Einaudi, 1990a.

Raggio, O., Etnografia e storia politica. La faida e il caso della Corsica, Quaderni Storici, 1990b, 75, pp. 937-954.

Rousseaux, X., Ordre moral, justices et violence: l'homicide dans les sociétés européennes. XIII ${ }^{\mathrm{e}}$ $\mathrm{XVIII}^{\mathrm{e}}$ siècles, in Garnot, B. (Dir.) Ordre moral et délinquance de l'Antiquité au XXe siècle, Actes du colloque de Dijon, 7 et 8 octobre 1993, Dijon, Éditions Universitaires de Dijon, 1994, pp. 65-82.

Rovere, A., Violence sociale et État royal dans la Corse du XVIII ${ }^{e}$ siècle, in Coll., Banditisme et violence sociale dans les sociétés de l'Europe méditerranéenne, Études corses, Ajaccio, La Marge Édition, 1995, pp. 261-267.

Salvini D. G., Giustificazione della rivoluzione di Corsica e della ferma risoluzione presa da corsi di mai più sottomettersi al dominio di Genova, Stamperia della Verità, Corte, 1758.

Serpentini, A. L., Bonifacio une ville génoise aux Temps Modernes, Préface d'É. Le Roy Ladurie, Ajaccio, Éditions La Marge, 1995.

Serpentini, A. L., La Coltivatione Gênes et la mise en valeur agricole de la Corse au XVII ${ }^{\mathrm{e}}$ siècle, Ajaccio, Éditions Albiana, 2000a.

Serpentini, A. L., Un littoral sous influence: la Corse sous la domination génoise à l'époque moderne (1562-1768), in Le Bouëdec, G., Chappé, F., (Dir.) Pouvoirs et littoraux du XVe au XXe siècles. Actes du colloque international de Lorient (24-26 septembre 1998), Université de Bretagne Sud Presses, Universitaires de Rennes, 2000b, pp. 211-223.

Wilson, S., Vendetta et banditisme en Corse au dix-neuvième siècle, édition originale: Cambridge University Press, 1988, Édition française: Ajaccio, A Messagera/Albiana, 1995.

\section{ANNEXES}



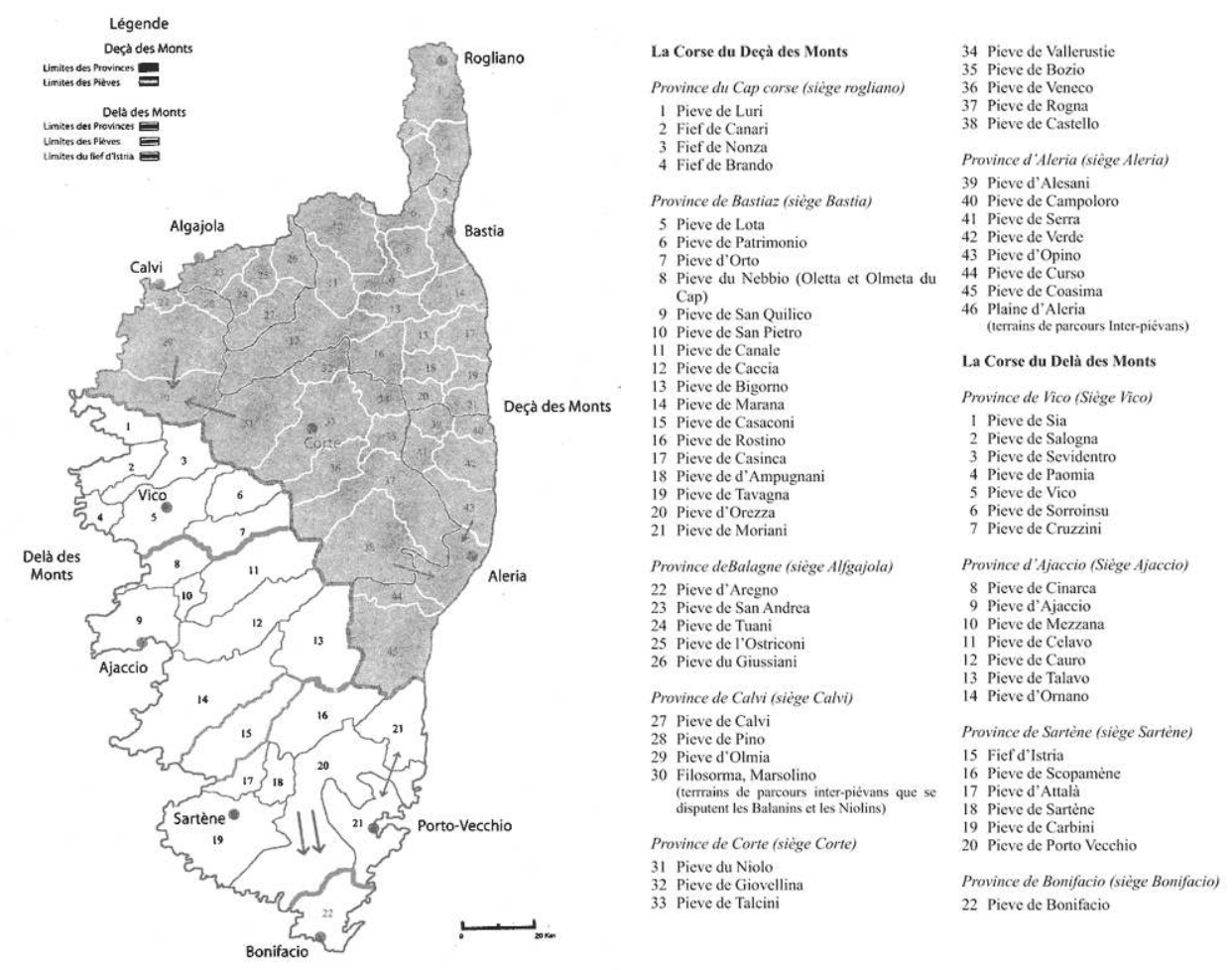

\section{NOTES}

2. Salvini, (1758).

3. Répondant ainsi aux vœux réitérés d'une grande partie de la population et présentés par ses représentants, les Nobles Douze et les Nobles Six.

4. Natali, $(1736,1983)$. S'appuyant sur un opuscule intitulé Ragguali de' tumulti seguiti in Corsica, daté de 1731 et signé d'un pseudonyme, mais qu'il attribue à la propagande génoise, le Chanoine Natali est le premier à accréditer l'idée, tout en s'en indignant, que « d'une année sur l'autre on en comptait (les homicides) jusqu'à 900, presque tous impunis » p.139.

5. Selon les données présentées par Borlandi, (1942), cf. plus particulièrement, pp. 202-204, le tableau $n^{\circ} 2$ intitulé Stato della popolazione dell'Isola di Corsica prima del 1732 (1726 ?). À cette dernière date, la population de la Corse du Deçà des Monts dépasserait légèrement les 87000 âmes et celle de la Corse du Delà des Monts atteindrait les 35000 âmes. Soit au total environ 122000 habitants, chiffre qui, nous semble-t-il, doit être considéré comme une estimation plancher.

6. Dupâquier, (1988, T2, p. 68).

7. Le Roy Ladurie, (1975, p. 548).

8. D'après les statistiques que nous avons établies, la mortalité féminine représentait respectivement $2,90 \%$ des meurtres commis durant la période $1692-1702$ et 3,16\% pour les années 1702-1720.

9. Comme en témoignent nos travaux sur la population bonifacienne voir Serpentini (1995), et les nombreuses monographies réalisées par nos étudiants dans le cadre de mémoires de maîtrise ou de DEA. Et cela malgré le fait qu'une forte émigration masculine, mise en évidence par un de nos doctorants, draîne à cette époque un grand nombre de jeunes corses vers les armées italiennes, françaises et espagnoles.

10. Acinelli, (1770, 1994). 
11. Le dernier en date, Francis Pomponi justifie la décision génoise d'interdire les armes à feu par « ... la volonté de remédier au délabrement d'un pays où l'on compte plus de 900 meurtres par an... », cf. Pomponi (1995, p. 310).

12. Ibidem, p. 548.

13. Par Francis Pomponi, 1995, p. 298 et, dans le même collectif, par Ange Rovere (1995, p. 264).

14. Pour ce qui concerne plus particulièrement Gênes à l'époque moderne, à propos d'une enquête menée sur les archives utiles à une histoire de la criminalité dans les territoires de la République, la métropole, et les diverses circonscriptions administratives de Terre ferme, Carola Ghiara déplorait dès 1979 le fait que : « Le serie sono disordinate e lacunose » in Ghiara, (1979, p. 603).

15. Ibid., liasse 634 .

16. Ibid., liasse 662 .

17. Ibid., liasse 647.

18. Ibid., liasses 648 à 661 .

19. .Ibid., liasse 668.

20. Cette procédure n'est pas spécifique à la Corse. Elle découle de la volonté manifestée par Gênes, dès la fin du XVIe siècle et surtout durant le XVIIe, de contrôler la criminalité et d'organiser la justice criminelle partout sur son territoire. D'où la nécessité de faire remonter les informations dans ce domaine jusqu'aux instances dirigeantes. Osvaldo Raggio voit dans cette démarche, un des axes du renforcement et de la légitimation de l'état génois (1990, a, p. 7). C'est dans ce contexte, comme en témoigne une directive datant des années 1660-1670, qu'il sera fait obligation au gouverneur de Corse sortant - tout comme à ses homologues, responsables de territoires de terre ferme - de faire parvenir à Gênes un état détaillé des crimes de sang commis dans sa juridiction durant son mandat. A.S.G. Archivio Segreto - Propositionum, Liasse 1040.

21. À savoir, pour le delà des Monts les provinces de Vico, Ajaccio, Sartène et Bonifacio et pour le deçà des Monts les provinces du Cap Corse (siège Rogliano), de Calvi, de Balagne (siège Algajola), de Corte et d'Aléria. La province de Bastia était administrée directement par le gouverneur qui résidait dans cette ville. Ces lieutenants et commissaires qui, sous l'autorité du gouverneur, exercent un pouvoir de haute justice, sont, comme ce dernier, communément désignés par le terme de giusdicente.

22. Après y avoir joint son propre rapport concernant la province de Bastia, le gouverneur se contentait de procéder à un simple comptage de l'ensemble des meurtres en les ventilant selon leur nature et il adressait le tout, accompagné d'une note très brève, au Magistrato di Corsica.

23. Comme peut le laisser envisager l'assez grande diversité des résultats, calculés par périodes de deux ans, et surtout la pointe enregistrée dans les années 1704-1706 (223 cas), qu'il convient cependant de relativiser car elle est essentiellement provoquée par la montée spectaculaire de décès accidentels (45) et des éliminations de bandits (23), $c f$. tableau $n^{\circ} 2$.

24. Il s'agit des années 1690-1694, 1702-1708 et 1710-1720.

25. Statistiques présentées par Xavier Rousseaux (1994, p. 70).

26. Bayard (1972, p. 273).

27. Depuis qu'elle a définitivement pris, au XVIe siècle, le relais de la pieve.

28. À elles deux, sur la base de deux tiers pour Bastia, un tiers pour Ajaccio, elles concentrent à peu près le dixième de la population insulaire.

29. Graziani, Stromboni (2000).

30. Serpentini (2000, a).

31. Serpentini, 2000a.

32. Cf. plus particulièrement la synthèse de Benoît Garnot (1992, p. 27).

33. De ce point de vue, l'action du gouverneur Girolamo Veneroso, est particulièrement explicite. En charge de la Corse dans les années 1706-1708, il se distingua par une très grande fermeté, et un esprit de justice apprécié de tous. Pendant son mandat, les morts violentes diminuent de 
façon très significative. D'ailleurs, Veneroso, qui fut incontestablement un des plus talentueux gouverneurs de Corse mais dont la modestie n'était pas la qualité principale, se fait gloire de ces résultats auprès du Magistrato di Corsica, dans une lettre en date du 16 avril 1708 qui accompagne la liste des homicides commis pendant le deux dernières années: [Accliuse ritroveranno Vostre Signorie Serenissime, in seguito di vostri stimatissimi commandi le note degl'omicidij successi in questo Regno /.../ stimo che difficilmente si troverà altro governo, in cui siano meno. Ne riconosco il vantaggio dall'assistenza di nostro Signore Iddio, che spero me la continuerà per il poco tempo che mi resta di servire qui le Vostre Signorie Serenissime], A S G, Fonds Corsica, liasse 649. « Faisant suite à vos estimables recommandations, vos seigneuries sérénissimes trouveront, ci-joint, le relevé des homicides survenus dans ce royaume /.../j'estime qu'il sera difficile de trouver un gouvernement où il s'en sera produit moins. Je reconnais le devoir à l'aide de notre Seigneur Dieu, et j'espère qu'il continuera à me la prodiguer pour le peu de temps qu'il me reste à accomplir ici, au service de vos sérénissimes Seigneuries ».

34. Pour cette période, qui correspond au mandat du Gouverneur Pietro Francesco Fieschi, 45 décès accidentels sont relevés, dont 26 pour les seules provinces de Bastia (13) et d'Aléria (13). Les causes des décès accidentels survenus dans les provinces de Bastia et d'Ajaccio (3 cas) ne sont pas mentionnées. Pour les autres provinces, les décès accidentels se répartissent comme suit: chute d'un arbre : 6 cas, rupture d'une branche $: 3$, noyade : 7, travaux agricoles 3 , autres : 10. La très grande majorité des noyades se produisent aux mois de mars - avril à une époque où de fortes pluies ajoutent leurs effets à la fonte des neiges et transforment le moindre ruisseau en torrent impétueux. Les décès provoqués par des chutes d'arbres ou par la rupture de branches sont étroitement liés au cycle des récoltes. Au mois de juin, la chute d'un cerisier se révèle souvent mortelle, car ces arbres, rarement taillés, sont généralement très hauts. Au mois d'octobre-novembre, les paysans paient tribut au châtaignier dont les branches chargées de fruits cèdent parfois sous l'effet du vent. A S G, Fonds Corsica, liasse 648.

35. ... piuttosto per disgrazia che per altra fine, come dà gli atti - A S G, Fonds Corsica, liasse 658.

36. Nous renvoyons à une abondante littérature d'où ressortent deux ouvrages principaux, à savoir : Busquet (1994), et Wilson $(1988,1995)$.

37. 499 meurtres sur les 799 survenus entre 1704 et 1720 sont effectivement datés.

38. Muchembed (1989, p. 27).

39. A.S.G., Fonds Corsica, liasse 648.

40. Ibid., liasse 648 .

41. Ibid., liasse 658 .

42. Ibid., liasse 648 .

43. Ibid., liasse 649 .

44. Ibid.

45. A. S.G., fonds Corsica, liasse 658.

46. Cf. Serpentini (2000, a), pp. XIX-XX, et 137-164.

47. A. S.G., fonds Corsica, liasse 658.

48. À propos du peu de cas que l'on faisait, à l'époque, de la vie d'une femme, l'officier du régiment de Picardie écrivait en substance: "Avant l'arrivée des Français, il était très commun d'entendre dire qu'un tel venait de tuer sa femme. Quand cela arrive, et c'est toujours par jalousie, ils vont dire eux-mêmes à ses parents d'aller faire enterrer leur fille, morte à tel endroit; et ils sont persuadés qu'elle s'est mise dans le cas de mériter une telle mort. Aujourd'hui que la justice poursuit tous les crimes, on n'entend plus parler de ces horreurs ", in Caraffa (Ed. 1889, p. 207).

49. A. S.G., fonds Corsica, liasse 658.

50. A. S.G., fonds Corsica, liasse 654.

51. Il faut aussi dénoncer le rôle néfaste joué par les femmes dans le déclenchement des vendette à travers la pratique du "rimbecco » qui visait par des reproches permanents à inciter les hommes de leurs familles à exercer la vengeance. Ce délit était d'ailleurs sévèrement puni aux 
termes de l'article XIX des Statuts Criminels de Corse, amendé en 1581. Ainsi, c'est en application de ces dispositions que, le 3 juin 1705, la femme d'Anton Gio de la Serra, qui par rimbecco avait conduit son mari à tuer Anton Marco de Sant'Andria d'Attallà, sera condamnée par contumace à trois années de relégation sur l'île de Capraia (A. S.G., fonds Corsica, liasse 648).

52. Tous les observateurs français du XVIIIe siècle, de l'officier du régiment de Picardie à l'abbé Gaudin (Voyage en Corse et Vues politiques, Paris, 1787), ont lourdement insisté sur le comportement belliqueux du clergé insulaire dont les membres sont décrits comme étant ignares, de mœurs dissolues et trop souvent armés jusqu'aux dents. À la lumière des études les plus récentes, le trait semble assez forcé. Cependant il est intéressant de relever que, lors de la visite pastorale ordonnée en 1740 par Monseigneur Agostino Saluzzo, évêque de Mariana d'Accia, le visiteur général s'ingénia à classer, pour chaque paroisse, les clercs en trois catégories : ceux qui portaient des armes ou qui les avaient portées lors des dernières Révolutions et enfin de ceux qui s'en étaient toujours abstenus in Caraffa (1890, pp. 131-238). Par ailleurs, il faut noter que les membres du clergé insulaire payaient eux aussi un lourd tribut à la violence. Plusieurs d'entre eux furent assassinés durant la période considérée, et non des moindres, comme par exemple Giovan Battista Mattarana de Bonifacio, tué à coups d'épée et de poignard par les frères Lorenzo et Giuseppe Maria Celani le17 juin 1719, ou encore Rocco Serra, abattu dans la campagne par un tir d'arquebuse le 11 janvier 1720; A. S.G., fonds Corsica, liasse 661.

53. A. S.G., fonds Corsica, liasse 660 .

54. Coppolani, Serpentini (1998). Cf. en particulier les articles XXXII et XXXIII, intitulés respectivement De l'homicide et Des poisons.

55. Leggi nuove et Addizioni fatte agli Statuti di Corsica, textes publiés à la suite des Statuts par Gio. Carlo Gregorj ( 1843).

56. Cela, essentiellement en application du décret approuvé, le 25 mai, le 15 juin et le 20 novembre 1635, par diverses instances de l'État génois et intitulé Legge contro coloro che ammazzano sotto pace o per vendetta. Retenons qu'on procédait de même dans le domaine péninsulaire de la République. Cf. Raggio (2000,a, p. 12-13).

57. Mais parfois aussi grâce à l'intervention des familles des assassins qui entendent mettre fin à une vendetta par trop pénalisante. Quel que soit le cas de figure retenu, la famille demeure par essence un intermédiaire incontournable, et ce aussi bien dans le domaine péninsulaire qu'en Corse. Cf. sur ce thème Raggio (1990, a et b).

58. En dernière analyse, si une certaine évaporation, due à la dissimulation, au manque de confiance envers la justice et au désir de régler soi-même ses comptes, a pu affecter ce « chiffre noir » de la criminalité de sang, son impact - difficile à estimer - n'a pu, cependant, qu'être marginal. En effet, en application de l'Article II des Statuts criminels, les podestats des communautés rurales étaient tenus, sous peine d'amende, de faire connaître rapidement au commissaire ou au lieutenant de la province, tous les délits, et donc, a fortiori, les homicides commis sur le territoire de leur juridiction. D'ailleurs, en cas de non-observation de ces directives, il semble évident que la dérive vindicative, inéluctable, aurait immanquablement attiré l'attention de l'autorité de tutelle, très sensibilisée à ce problème.

59. Elles sont extraites de la série Rollorum Partitarum du fonds Corsica de l'Archivio di Stato de Gênes, liasses 1085, 1126, 1164.

60. Il est vrai que si des désordres importants se produisaient dans l'intérieur des terres, les forces du bargello pouvaient compter sur le concours des troupes stationnées dans les présides. Cf. Serpentini (2000b). Ajoutons que ces agents de la force publique, que l'on appelait indifféremment famegli ou sbiri, souffraient d'une fort mauvaise réputation, apparemment méritée. Particulièrement turbulents, ils sont non seulement à l'origine de la majorité des duels répertoriés durant cette période, mais ils se livrent aussi aux pires exactions, souvent avec l'accord tacite de l'administration de tutelle. Ainsi, dans les provinces, on les voit parfois 
s'installer, à la demande de créanciers, dans les demeures de leurs débiteurs et s'y faire entretenir tant que ces derniers n'ont pas réglé leurs dettes.

61. Cette procédure n'est pas une spécificité génoise. Il en va de même comme l'a démontré Robert Muchembled, des lettres de rémission accordées par les souverains espagnols, qui «en l'absence de forces policières suffisantes et d'une répression qui serait efficace /.../ assument une lente transition entre le temps de la vengeance privée et celui de l'obéissance à l'état absolu... » (1989, pp. 22-23). Mais ce qui pose problème c'est qu'en Corse cela est devenu une réponse trop fréquente, quasiment systématique. Ainsi, un exemple parmi tant d'autres, dans la province de Sartène sur les six meurtriers recensés durant le biennio 1714-1716, un n'a pas encore été jugé; les cinq autres ont été condamnés par contumace, et quatre d'entre eux ont bénéficié de l'indult; A SG, fonds Corsica, liasse 656.

62. Clairement énoncé, dans un rapport sur le maintien de l'ordre et l'exercice de la justice adressé au Magistrato di Corsica, par le gouverneur Pietro Francesco Fieschi, peu avant sa sortie de charge, le 22 mars 1706 : " giachè il proverbio, a ponto di qui comprovato dall'esperienza come tutti gl'altri proverbij, dice che se il Corso non piglia il Corso, niun'altro piglia il Corso ". "D'après le proverbe, conforté, comme tous les autres, par l'expérience, qui affirme que si un Corse ne peut s'emparer d'un autre Corse, personne d'autre n'y parviendra »; A S G, fonds Corsica, liasse 648. C'est sans doute en application de ce principe et pour utiliser ces compétences que Gênes, dans son territoire de terre ferme, engage fréquemment les troupes corses dans la répression du banditisne. $C f$. Raggio (1990a).

\section{RÉSUMÉS}

Utilisant les rôles de la mortalité violente, tenus à jour par les gouverneurs génois en charge de la Corse à la fin du XVII ${ }^{e}$ siècle et durant les deux premières décennies du siècle suivant, cette communication permet de cerner avec précision l'impact de la criminalité de sang. À cette époque, avec un taux annuel de mortalité par homicide s'élevant à 45,3 pour 100000 habitants, l'île comptait incontestablement parmi les régions les plus criminogènes de l'Europe occidentale. Toutefois, pour aussi effrayants qu'ils soient, ces chiffres sont bien en deçà de ceux qui ont été véhiculés par toute une tradition historiographique. Après avoir clairement mis cela en exergue, l'auteur s'attache à étudier les différents aspects de cette criminalité - la nature des homicides, l'impact microrégional, les saisons et les armes de la mort, etc.- en relation avec les mesures prises par la République de Gênes pour essayer de la juguler.

Using the registers of violent deaths kept by the Genoese governors in charge of Corsica at the end of the 17th century and during the first two decades of the 18th century, this contribution neatly circumscribes the impact of homicide. In this period, with an annual homicide rate of 45.3 per 100,000 inhabitants, the island was indisputably one of the most crime-ridden regions of Western Europe. However frightening these figures may be, though, they are well below those reported by a whole historiographical tradition. Having made this clear, the author turns to the different aspects of this kind of crime : the nature of the homicides, their impact on the microregion, the seasons and the weapons chosen, etc., which he relates to the steps taken by the Republic of Genoa to attempt to curb such crimes. 


\section{AUTEUR}

\section{ANTOINE LAURENT SERPENTINI}

Université de Corse, Avenue Jean Nicoli, 20250 CorteAntoine Laurent Serpentini est professeur d'histoire moderne à l'Université de Corse. Il a notamment publié : Bonifacio une ville génoise aux Temps Modernes, Préface d'É. Le Roy Ladurie, Ajaccio, Éditions La Marge, 1995; la Coltivatione Gênes et la mise en valeur agricole de la Corse au XVII ${ }^{e}$ siècle, Ajaccio, Éditions Albiana, 2000; Sources notariales, confrontations économiques, stratégies matrimoniales et patrimoine à Bonifacio dans la deuxième moitié du XVIIIe siècle, Mélanges de l'École Française de Rome Italie et Méditerranée, 2000, 112, 1, pp. 253-279. Il est codirecteur de la collection, Les grands textes du droit corse (dont il a signé le $n^{\circ} 3$ avec J.-Y. Coppolani - Sept volumes prévus) et dirige le projet d'édition d'un Dictionnaire historique de la Corse, auquel collaborent plus de cent universitaires français et étrangers. 